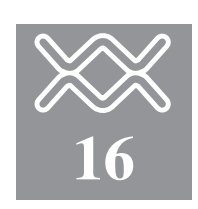

\title{
Trasferimento dei nuovi mat
nella clinica quotidiana: vantaggi e limiti
}

\author{
C. Campisi \\ CNR - Istituto Ingegneria Biomedica, Ospedale San Pietro, Fatebenefratelli, Roma
}

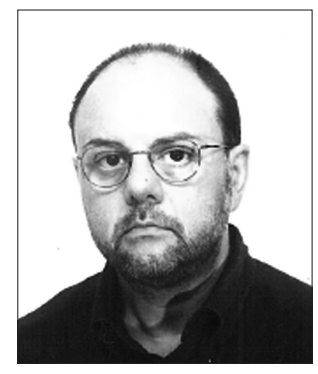

$\mathbf{E}$ mezzo secolo fa, nel 1956, il premio Nobel per la medicina veniva assegnato a uno sconosciuto urologo di un piccolo paese della Germania che, appena laureato, aveva eseguito su se stesso un cateterismo cardiaco attraverso una vena del braccio e utilizzando un catetere ureterale. Per queste esperienze il dottor Werner Theodor Forssmann era stato allontanato dalla Clinica del famoso professor F. Sauerbruch (che pure si sarebbe compromesso con alcune esperienze condotte con le SS nei lager nazisti) perché troppo cruente.

Ebbene, dopo mezzo secolo, da un lato la maggior parte dei problemi sono stati superati, bisogna pure ammettere dall'altro che molte altre problematiche cliniche devono ancora essere affrontate, prima che risolte, nella maniera più incisiva e corretta: tassi di complicanze troppo alte, troppo costose e invalidanti, quelle infettive in testa, procedure di impianto ancora troppo rischiose, modalità di gestione troppo personalistiche: come conciliare tutto questo?

Di fatto si tratta di metodologie che an- cora oggi in molti ambienti medici, e soprattutto per i presidi a lungo termine, vengono considerate importanti, ma non sempre fondamentali, utili, ma potenzialmente pericolose, soluzione di alcuni problemi, ma causa di altri, e ogni insuccesso viene additato a conferma di pregiudizi spesso difficili da eradicare.

Non si tratta di una realtà italiana: in molte aree di Paesi stranieri il catetere a lungo termine è utilizzato in maniera sporadica, ma in ogni parte d'Europa un dato appare evidente: dove c'è più cultura del problema il mercato è più vasto di dove l'approccio è più diretto e meno complesso.

Dal punto di vista dei materiali gran parte del problema deve essere attribuita al fatto che la ricerca nel settore è minima, semplicemente perché strutturalmente considerata a bassa tecnologia e a basso costo, e quindi tutte le migliorìe derivano di fatto dalla estensione di altre applicazioni (ad esempio, il silicone e i poliuretani dei cateteri) o da applicazioni intuitive e legate al semplice "buon senso" (forme e superfici dei presidi, kit di impianto...).

Anche laddove le possibilità di ritorno economico sembravano più importanti, come nel campo dei cateteri trattati con sostanze antinfettive e antitrombotiche, gli investimenti sono stati poco incisivi e le metodologie approssimative e spesso già superate.

Quanto ora brevemente descritto viene però comunemente e diversamente interpretato (a nostro avviso in maniera riduttiva perché riferita a una conoscenza poco approfondita delle problematiche coinvolte) in questi termini: la metodologia attuale è sufficientemente matura e definita nei suoi vantaggi e limiti e quindi per certi versi "chiusa", cioè difficile a possibilità di evoluzione.

Di fatto, oggi abbiamo a disposizione molti presidi, con possibilità e caratteristiche di scelta apparentemente molto ampie, ma c'è da chiedersi se tutte queste possibilità di scelta siano in grado di soddisfare appieno le richieste di chi li utilizza. Di contro l'esperienza quotidiana mostra che ogni operatore pratica pochissime scelte all'interno dei listini offerti dalle aziende del settore.

\section{Cateteri e tecnologie di posizionamento}

Per i presidi a medio e lungo termine si devono affrontare alcune problematiche, tutte correlate al momento del posizionamento del catetere. La procedura, proprio perché teoricamente procrastinabile e programmabile, deve essere affrontata e concentrata in pochi 


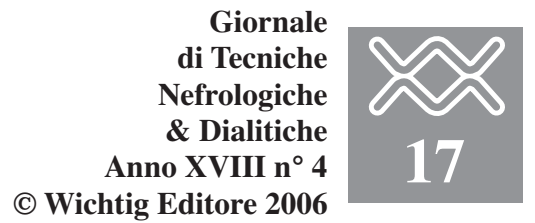

Centri selezionati, o può essere diffusa in tutti i Centri clinici che hanno in cura malati cronici, nefropatici e tumorali in particolare?

Il problema è giustificato dall'alto tasso di morbilità, molto più alto che in altre pratiche chirurgiche ben più complesse. Nel caso in cui si crederà alla concentrazione della metodica in pochi Centri, allora l'adozione dell'ecografo dedicato, della guida Doppler, degli ambienti dedicati e delle attrezzature più evolute sarà necessaria e indispensabile, oltre che utile. In caso contrario, si potrà fare ricorso prevalentemente a tecnologie più collaudate e disponibili, come l'amplificatore di brillanza, o a più basso costo, come la guida elettrocardiografica.

Si tratta, a nostro avviso, di comodità più che di indispensabilità. Per l'utilizzo della vena succlavia l'ecografo ha limiti noti e vantaggi irrilevanti per una metodica caratterizzata da percentuali di successo della puntura del 95-98\%, mentre per la puntura giugulare la comodità è evidente fino a diventare importante per pazienti particolarmente obesi o dispnoici o con patologie associate debilitanti.

Riteniamo che un ecografo dovrebbe essere reso disponibile in tutte le sale operatorie in cui si attui una chirurgia maggiore e per questo non sarebbe difficile ricorrere al suo impiego per il cateterismo di pazienti particolari da parte di chirurghi, anestesisti o nefrologi. Ma riteniamo che l'acquisto di una attrezzatura dedicata rappresenti uno spreco di risorse rispetto a una analoga più versatile.

Per la valutazione della posizione della punta del catetere riteniamo che la guida elettrocardiografica mediante elettrodo interno rappresenti una metodica efficace, a basso costo, efficiente. Ma perché viene così poco utilizzata? Evidentemente per praticità, perché non è resa disponibile da aziende impegnate a rivolgere la propria attenzione a soluzioni più costose.

In ogni caso, da parte dell'operatore devono essere operate le opzioni giuste, nel differenziare alcuni requisiti minimi irrinunciabili (ad esempio, il catetere deve essere opportunamente graduato, con inchiostrazioni chiare e numericamente progressive) mentre altri devono essere considerati secondari (come la rastrematura alla punta).

Altre scelte devono essere ponderate: ad esempio, potrebbe essere forse preferita l'adozione di un presidio valvolato sostituibile, monouso e a basso costo (needle-less systems) piuttosto che quella di una valvola integrata, con tutti i pregi e i difetti, nel catetere (catetere di Groshong).

Sulla scelta del silicone o del poliuretano (laddove non siano necessarie specifiche necessità di flusso) e sulla preconnessione alla porta ciascuno può mantenere i propri giudizi, essendo sicuro di non poter essere smentito (ma neanche confortato) sulle proprie convinzioni.

Relativamente alla scelta del port, deve essere pretesa dall'azienda prescelta la possibilità di poter optare tra diversi profili, da adattare alla configurazione e alle necessità del malato. La camera interna deve essere preferibilmente in titanio, perché i materiali plastici adottati (di solito di qualità strutturalmente inferiore rispetto a quanto impiegato per i cateteri) possono interagire (vedi ftalati) con alcuni farmaci veicolati e possono venire danneggiati dall'azione della punta dell'ago di Huber.

\section{Cateteri e infezioni}

Sul piano delle complicanze, non sussiste alcuna evidenza di superiorità tra un materiale e l'altro nel prevenire infezioni e trombosi del vaso. In particolare, il silicone potrebbe essere considerato teoricamente migliore rispetto ai poliuretani, in virtù di una minima reattività chimica delle superfici, ma tutto ciò non sembra essere, sul piano pratico, di alcuna importanza clinica.

Sul piano della struttura progettuale dei presidi bisogna considerare che ogni irregolarità delle superfici favorisce il ristagno di sangue e batteri, consentendo le migliori condizioni di replicazione di questi ultimi, che si troverebbero nelle condizioni nutrizionali e di temperatura ideali. In questo senso la camera del port costituisce un ambiente da sottoporre a pulizia con maggiore attenzione dopo l'utilizzo, con flushing pulsato attuato con siringhe di almeno $10 \mathrm{~mL}$.

Sempre sul piano teorico, maggiori sono le superfici esposte e maggiore è il rischio di infezione: ecco perché favorire cateteri di minor diametro e dal minor numero di lumi.

Per quanto riguarda i cateteri trattati con sostanze antibiotiche o antisettiche, al di là di linee guida predisposte sulla base di studi limitati e con metodologie spesso discutibili e talvolta non indipendenti, riteniamo che non sussistano motivazioni sufficienti per raccomandarne l'uso routinario, soprattutto se a compensazione di tecniche di posizionamento e di gestione carenti. Non è inutile, a tal proposito, ricordare che l'azione antibatterica (e comunque non antifungina e antivirale) viene attuata da questo tipo di presidi nel periodo immediatamente post-impianto, quando il tasso di complicanze infettive è di regola basso.

Riteniamo invece che un maggior controllo possa derivare da tutte quelle procedure di misurazione della qualità del lavoro prodotto come il monitoraggio delle infezioni correlate al catetere e delle procedure di gestione.

È però comprensibile, di fronte a procedure complesse, che le novità del mercato siano state indirizzate verso scelte più immediate, anche se economicamente più onerose, ed in questo contesto crediamo di poter fare questa analisi.

Per l'accesso venoso centrale a breve termine l'abilità dell'operatore sarà ancora chiamata a supplire alla semplicità delle metodica che, in un contesto di urgenza clinica come nei reparti di emergenza, deve essere di pronto impiego. Tale competenza viene ormai unanimemente ritenuta bagaglio culturale della professionalità e quindi il problema non deve essere posto se non nei termini della assoluta necessità di semplificare le manovre in sede di posizionamento. In questa direzione la raccomandazione è quella di rivolgere la propria attenzione alla completezza e alla efficienza del kit di posizionamento percutaneo: una affilatura ottimale dell'ago, la disponibilità di telini sterili adeguati (ma non solo!) potrebbero ad esempio fare la differenza. 
Per i presidi a medio-lungo termine il controllo delle infezioni dovrà essere attuato attraverso le migliori scelte dei materiali in funzione dell'utilizzo: ad esempio è inutile utilizzare un catetere tunnellizzato per neoplasie solide che ne richiedono l'impiego intermittente, o al contrario, è controproducente posizionare un ago di Huber a permanenza in port che devono sopportare a lungo terapie infusionali.

\section{Cateteri e trombosi}

Fino alla fine degli anni Settanta proprio la trombosi centrale aveva impedito la diffusione della metodica.

Gli studi di base più importanti risalgono agli anni Ottanta, e da allora è chiaro che la complicanza è direttamente proporzionale al diametro del catetere, considerando come costantemente bassa la pressione venosa centrale. È intuitivo che il diametro del catetere deve essere rapportato al diametro della vena che lo ospita, e tale osservazione è utile nel considerare le vene del braccio.

La diretta conseguenza di tutto ciò è che alcune metodiche, come quelle dedicate alla dialisi, sono intrinsecamente gravate da tassi di complicanze trombotiche più alte. In questo campo particolare sarà necessario considerare anche le pressioni attuate dalle macchine dialitiche, trasmesse indirettamente alla parete della vena attraverso il catetere e l'impiego di eparina durante la dialisi stessa.

Un altro aspetto importante è dato dalla rigidità e dalla durezza del catetere, espressione non solo del materiale utilizzato (il silicone e gli ultimi poliuretani sono ormai quasi equivalenti) ma anche degli spessori (maggiori per il silicone) e di eventuali setti interni nel caso di cateteri multilume.

Per i cateteri a due lumi come quelli impiegati nella dialisi sarebbe meglio scegliere presidi con modalità di sepimentazione dei lumi che tenga conto delle possibilità torsionali in funzione delle curve di stazionamento e delle vibrazioni trasmesse dalla pulsazione cardiaca. Per questo motivo sarebbe forse meglio utilizzare, ove possibile, cateteri a forma già predisposta, in grado anche di garantire le resistenze interne migliori ai fini del buon funzionamento della dialisi.

\section{CONCLUSIONI}

A conclusione di quanto brevemente esposto, ci sentiamo di raccomandare quanto segue:

1) Non avere pregiudizi e commisurare i propri giudizi con quelli di altri.

2) Non raccomandare ad altri le proprie esperienze se non sono espressione di dati oggettivi e oggettivamente validi.

3) Sottoporre ad analisi critica le esperienze riportate da altri, commisurandola alle proprie esperienze e adattandole alla realtà clinica abituale.

4) Elevare il bagaglio di conoscenze in modo tale da poter assumere scelte responsabili.

5) Essere pronti a modificare le proprie scelte in funzione delle mutate capacità e dei miglioramenti recepiti.

\section{BIBLIOGRAFIA}

1. Khare MD, Bukhari SS, Swann A, Spiers P, McLaren I, Myers J. Reduction of catheter-related colonisation by the use of a silver zeolite-impregnated central vascular catheter in adult critical care. J Infect 2006.

2. Gupta H, Araki Y, Davidoff AM, Rao BN, Hoffer FA, Billups C, Wu J, Shochat SJ. Evaluation of pediatric oncology patients with previous multiple central catheters for vascular access: Is doppler ultrasound needed? Pediatr Blood Cancer 2006.

3. Gersch MS. Treatment of dialysis catheter infections in 2004. J Vasc Access 2004; 5(3): 99-108.

4. Bruno S, Remuzzi G. Vascular access-related thrombotic complications: research hypotheses and therapeutic strategies. J Nephrol 2006; 19 (3): 280-5. 